\title{
Phonological encoding in the attentional blink paradigm
}

\author{
Veronika Coltheart and Lisa S. Yen \\ Macquarie University, Sydney, New South Wales, Australia
}

\begin{abstract}
The attentional blink refers to a deficit in reporting a second target that follows a first target within a few hundred milliseconds, when both targets occur in a sequence of distractors shown serially at rates of about 10 items per second. In four experiments, phonological similarity of post-Target 1 distractors impaired dual target report within the interval in which the attentional blink occurs. Similarity of letter targets had a smaller, less reliable effect on performance. Phonological similarity of letter distractors did not affect single target identification (Experiment 3), but it continued to impair dual target report (Experiment 5), even when the targets belonged to a different category - namely, digits. The results demonstrated that, not only targets, but also distractors are encoded phonologically, despite the fact that distractors are irrelevant and never have to be reported.
\end{abstract}

There are limits on attention to visually presented items, both when shown in briefly presented arrays or serially presented at high rates. With rapid serial visual presentation (RSVP), the first of two targets embedded in a sequence of irrelevant distractors is detected accurately but the second is frequently missed if it follows a few hundred milliseconds afterward. The deficit in second target report, termed the "attentional blink" by Raymond, Shapiro, and Arnell (1992), is transitory: Report improves as the interval between targets increases. Several studies (e.g., Chun \& Potter, 1995) also demonstrated that the attentional blink did not occur, or was reduced, if the second target (T2) immediately followed the first (T1), a phenomenon known as "lag 1 sparing." Additionally, Seiffert and Di Lollo (1997) showed that both metacontrast and pattern masking impaired $\mathrm{T} 1$ processing. These masking effects indicate that relatively low-level visual interference contributes to the deficit.

The relationship between targets and distractors is another factor modulating the size of the attentional blink. The blink is reduced if targets and distractors belong to different but familiar categories, for example, if targets are letters, and distractors are digits (Arabic numerals). If the targets are letters, but distractors are symbols (e.g., *, \#, +), the attentional blink is absent (Chun \& Potter, 1995). These findings implicate a higher level stimulus category relationship as a crucial determinant of the occurrence of the attentional blink. Although Maki, Bussard, Lopez, and Digby (2003) demonstrated that variation in featural differences between targets and distractors could explain findings attributed to categorical differences, it turns out that categorical differences between targets and distractors nevertheless can modulate the size of the attentional blink, even when feature differences are held constant (Dux \& Coltheart, 2005).
Consequently, the attentional blink is induced by both lower level visual and higher level central processing limitations. It has been assumed that, in this task, most, or possibly all, items are briefly identified (Stage 1), but that a second processing stage (Stage 2) is required to register the targets in reportable form in either working memory (Chun \& Potter, 1995) or visual short-term memory (Isaak, Shapiro, \& Martin, 1999). The attentional blink has been attributed to a bottleneck in communication from Stage 1 to Stage 2, this communication being assumed to involve slow serial processing (see, e.g., Chun \& Potter, 1995). To explain lag 1 sparing, these accounts had to assume that, when targets are temporally adjacent (lag 1), both can access Stage 2. Di Lollo, Kawahara, Ghorashi, and Enns (2005) argued that this explanation is somewhat ad hoc and demonstrated that, during $\mathrm{T} 1$ processing, a temporary loss of control over the attentional setting of the input filter increases interference by distractors.

Stage 2 processing was assumed to involve visual shortterm memory (VSTM) and registration in phonological working memory. Target-distractor visual similarity effects indicate involvement of VSTM (Isaak et al., 1999). Evidence for phonological coding in $\mathrm{T} 1$ report comes from Olson, Chun, and Anderson's (2001) finding of an increased attentional blink for polysyllabic $\mathrm{T} 1$ relative to a monosyllabic $\mathrm{T} 1$.

Considering now a different paradigm (list recall), the phonological similarity effect-inferior recall of phonologically similar items (words or letters), contrasted with recall of dissimilar items (see, e.g., Conrad \& Hull, 1964) - was interpreted as evidence of phonological coding. Furthermore, phonological similarity impairs recall even when lists are shown at high presentation rates of 8-9 items/sec (V. Coltheart \& Langdon, 1998) although

V.Coltheart, veronika@maccs.mq.edu.au 
the effect is smaller $(4 \%-6 \%)$ than with STM rates of presentation. Although the memory load is low in dual target identification, orthographic-to-phonological transcoding is required for oral target report. Even when report is written or typed, phonological-to-graphemic output coding is likely to occur. If phonological similarity of targets affects performance, that would suggest a role for phonological coding in the processing required for their report.

A further but related question concerns distractor processing. Report of distractors is not required in the dual target paradigm, so perhaps their representations must be actively inhibited. Hence, if phonological encoding is initiated for targets, it should not be initiated, or should be suppressed, for distractors. However, phonological encoding may be a process that, once invoked for a target, cannot be suppressed for distractors. Consequently, target report might be affected by whether distractors are phonologically similar to one another and to targets.

These possibilities were considered in Experiments 1 and 2 by manipulating phonological similarity of targets and distractors in an attentional blink task. In the experiments, we investigated whether there is any effect of (1) targets being similar or dissimilar to each other; (2) distractors being similar or dissimilar to each another; and (3) targets being similar or dissimilar to distractors. Because the post-T1 distractors are chiefly responsible for eliciting the attentional blink, the distractor similarity manipulation involved the four post-T1 distractors.

\section{EXPERIMENT 1}

\section{Method}

Subjects. Thirty-six psychology undergraduates participated for course credit.

Stimulus Materials. Two sets of six consonants having similar letter names (a shared vowel and similar distinctive consonant features) were selected. One set had names that ended with $/ \mathrm{i} /, \mathrm{B}, \mathrm{D}, \mathrm{T}$,

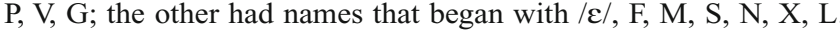
Similar target pairs were selected from these sets and embedded in sequences of 12 letters with no letter repeated. T1 occurred between Positions 3-6 and T2 followed at one of three lags $(2,3$, or 5).

Target pairs were phonologically similar (e.g., T, D) or dissimilar (e.g., N, D). In the similar distractor condition, four distractors following Target 1 were phonologically similar (e.g., B, P, T, D or F, M, S, L) or dissimilar (e.g., R, H, J, Q). At lags 2 and 3, some of these distractors followed T2. The other six distractors in the sequence were selected from 12 dissimilar letters of the alphabet (excluding $\mathrm{E}$ and $\mathrm{K})$. There were five conditions:

1. Targets and distractors all similar: $\mathrm{T} 1, \mathrm{~T} 2$, and four postT1 distractors all phonologically similar to one another (e.g., \#ORVDBTPGUYQI\&)

2. Targets similar and distractors similar: $\mathrm{T} 1, \mathrm{~T} 2$ similar (e.g., G, D), with the four post-T1 distractors similar to each other (e.g., F, M, S, X), dissimilar to targets (e.g., \#IA巨B

3. Targets similar and distractors dissimilar: $\mathrm{T} 1, \mathrm{~T} 2$ similar, with the four post-T1 distractors dissimilar to each other

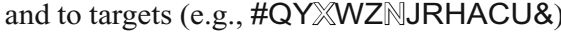

4. Targets dissimilar and distractors similar: $\mathrm{T} 1, \mathrm{~T} 2$ dissimilar (e.g., B, J), four post-T1 distractors similar to each other but not to targets (e.g., \#JCBNSWMFOQYZ\&)

5. Targets dissimilar and distractors dissimilar: $\mathrm{T} 1, \mathrm{~T} 2$ dissimilar, four post-T1 distractors dissimilar to each other and to targets (e.g., \#ZQXAGYCIHURJ\&)
Twelve trials of each condition occurred for each of three lags (2, 3, 5): 180 trials

Apparatus and Procedure. Sequences were presented on 700-MHz Apple eMac PowerPC G4 computers on 17-in. monitors programmed in PsyScript (Bates \& D'Oliveiro, 2003). The laboratory had normal room illumination with up to 5 subjects tested. Sequences consisted of 10 black and 2 white letters (targets) in uppercase Arial font (visual angle of $1.5^{\circ}$ ), preceded by a hashmark (\#) and finishing with an ampersand (\&). Each item appeared for $33 \mathrm{msec}$ on a gray background, followed by a 77-msec gray field. After each sequence, subjects typed target letters into a response box on the screen. They were informed that 2 white letters were presented, but that they could report fewer. A brief practice task preceded the experiment.

\section{Results}

Mean percentages of correct $\mathrm{T} 2$ conditional upon correct report of T1 (T2|T1) were calculated for each lag and each target-distractor condition. A repeated measures ANOVA and a planned contrast showed that target report was no worse when both targets and the four post-T1 distractors were all similar (Condition 1) than it was when the T1 and T2 were similar (e.g., T, D) but differed from the similar post-T1 distractors (e.g., F, X, S, N: Condition 2). Another ANOVA was conducted with target similarity (similar, dissimilar), distractor similarity (similar, dissimilar), and lag $(2,3,5)$ as factors, and the condition means are shown in Figure 1. (Only significant effects are reported throughout.) The effect of lag was significant $[F(2,70)=147.72$, $\left.M S_{\mathrm{e}}=294.1, p<.0001\right]$. Target report increased with lag. A trend analysis revealed a significant linear component $[F(1,70)=295.13, p<.0001]$. Distractor similarity reduced $\mathrm{T} 2 \mid \mathrm{T} 1$ report from $66.9 \%$ to $61.7 \%[F(1,35)=$ $\left.10.45, M S_{\mathrm{e}}=284.46, p<.005\right]$. The interactions between distractor similarity and lag $\left[F(2,70)=3.66, M S_{\mathrm{e}}=141.7\right.$, $p<.05]$ and between target similarity and lag $[F(2,70)=$ 3.91, $\left.M S_{\mathrm{e}}=143.8, p<.05\right]$ were significant. Planned comparisons showed that distractor similarity impaired performance only at lag $2[F(1,70)=23.44, p<.0001]$ and marginally at lag $3(p<.09)$. Target similarity impaired performance only at lag $3[F(1,70)=4.84, p<.05]$. T1 report averaged at $93 \%$ and did not vary significantly with lag; thus the effects were on $\mathrm{T} 2$ report.

\section{Discussion}

The T2|T1 scores showed clear evidence of an attentional blink with linear increase in performance across lags 2-5. Phonological similarity of T1 and T2 impaired performance at lag 3 only, and the impairment was small $(4.4 \%)$ relative to the effect of distractor similarity $(9.6 \%$ at lag 2). Distractor similarity reduced performance regardless of whether the similar distractors resembled targets. In fact, the condition of maximal similarity, Condition 1 (targets and distractors all similar to one another), yielded performance no worse than Condition 2 (targets similar and distractors similar with similarity differing between them). Thus, the results showed that phonological similarity of to-be-ignored distractors reduced target report, specifically for the second target during the attentional blink interval.

It seems that phonological activation of the names of visually presented letters automatically occurs for distrac- 


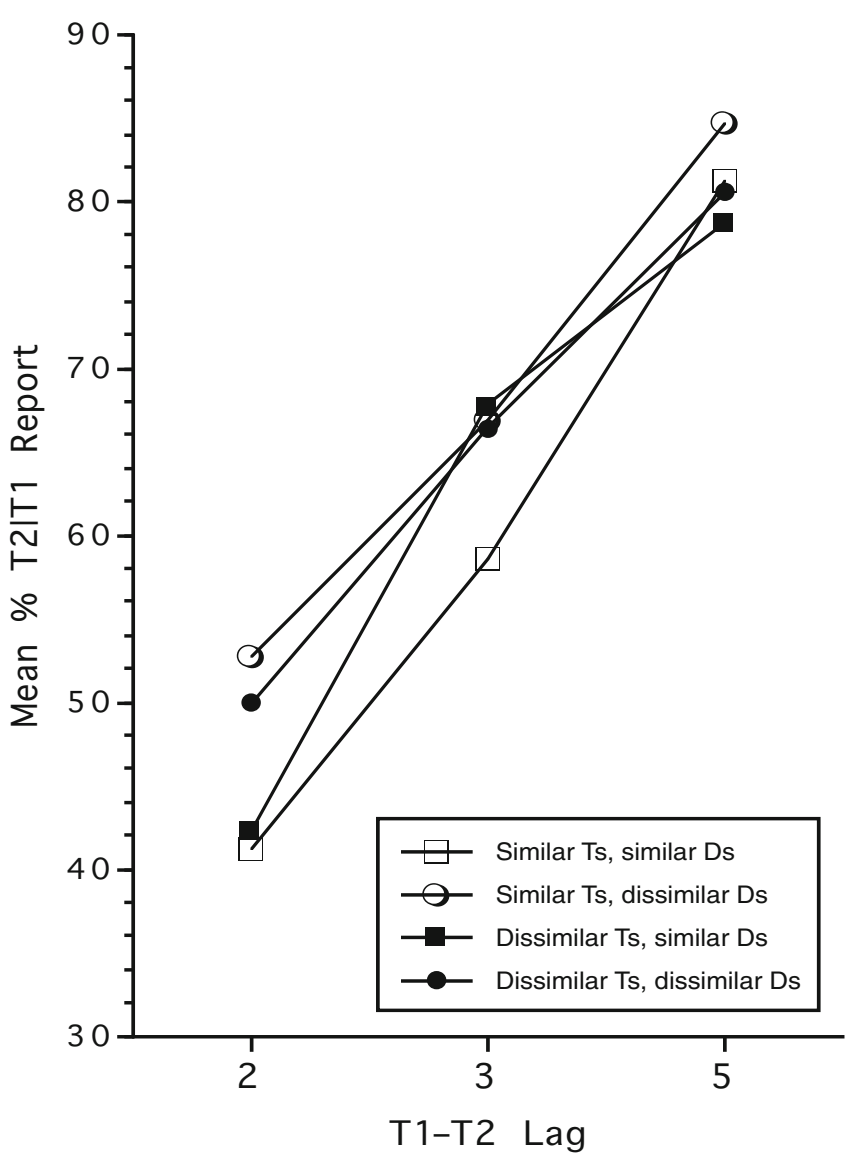

Figure 1. Mean percentages of correct T2 $\mid \mathrm{T} 1$ report for each of four conditions of target and distractor similarity as a function of T1-T2 lag for Experiment 1.

tors following T1. It happens even though report required no overt oral response (since targets were typed). Target report may rely on explicit phonological coding of items prior to response execution. When this process is initiated for T1, distractors are phonologically coded, too. The phonological encoding process cannot be suppressed for irrelevant items, at least not when these are also letters.

\section{EXPERIMENT 2}

Experiment 2 was conducted both to assess the replicability of the phonological effects and to examine target report at lag 1 when the blink is reduced or absent. If adjacent targets are easily encoded for report, phonological coding of distractors might more successfully be suppressed.

\section{Method}

Subjects. Thirty-four new subjects from the same pool participated.

Stimulus Materials, Apparatus, and Procedure. These were the same as in Experiment 1 except that lag 2 was replaced by lag 1 (with all four critical distractors following T2).

\section{Results}

The conditional $\mathrm{T} 2 \mid \mathrm{T} 1$ scores were subjected to ANOVAs, and a planned contrast indicated dual target report did not differ significantly when targets and distractors were all similar to each other (Condition 1) and when targets were similar and distractors were similar but similarity differed between them (Condition 2). After exclusion of Condition 1 , a 2 (target similarity) $\times 2$ (distractor similarity) $\times 3$ (lag) ANOVA was performed. The mean percentage T2 $\mid \mathrm{T} 1$ scores for each condition are shown in Figure 2. The following effects were significant: lag $\left[F(2,66)=22.18, M S_{\mathrm{e}}=512.1, p<.0001\right]$ and distractor similarity $\left[F(1,33)=9.28, M S_{\mathrm{e}}=206.2, p<.005\right]$. The effect of target similarity approached significance $\left[F(1,33)=3.86, M S_{\mathrm{e}}=138.5, p<.06\right]$. Trend analysis of the lag effect indicated significant linear and quadratic contrasts $[F(1,66)=16.15, p<.0005$, and $F(1,66)=$ 28.21, $p<.0001$, respectively]. Planned contrasts of the similarity effects indicated that target similarity significantly impaired performance only at lag $1[F(1,33)=$ $5.09, p<.05]$, whereas distractor similarity impaired per-

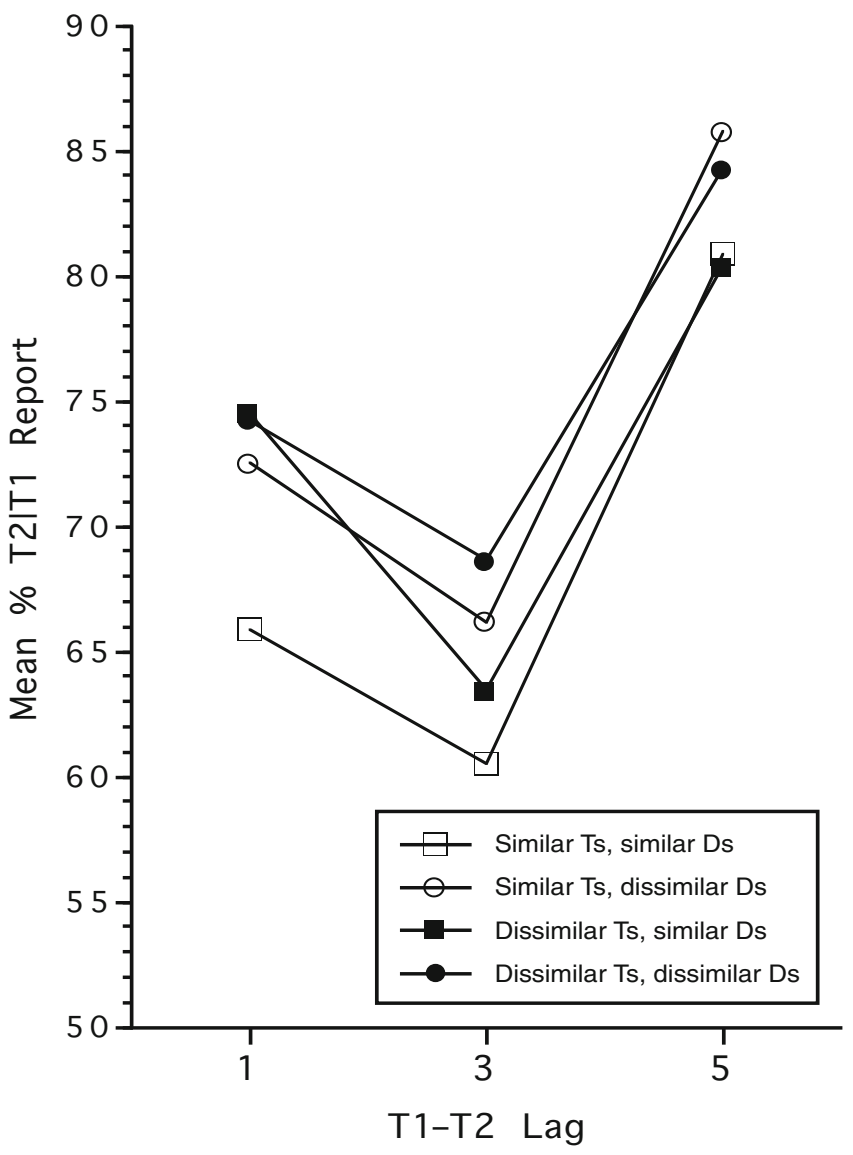

Figure 2. Mean percentages of correct T2 $\mid \mathrm{T} 1$ report for each of four conditions of target and distractor similarity as a function of T1-T2 lag for Experiment 2. 
formance at lag $3[F(1,33)=7.27, p<.01]$ and lag 5 $[F(1,33)=4.86, p<.05]$.

\section{Discussion}

Lag 1 sparing occurred along with an attentional blink manifest at lag 3 . The effect of target similarity was small and reliable only at lag 1 . The previous finding, that distractor phonological similarity reduces dual-target report, was confirmed. An effect of target phonological similarity can be expected, given that these items must be distinguished and consolidated for report. However, the phonological similarity effect exerted by distractors is surprising, because these are not to be recalled (although distractor intrusions occurred-especially the distractor after T2). The results indicate some degree of obligatory phonological encoding for irrelevant items following T1 in RSVP sequences.

\section{EXPERIMENT 3}

Experiment 3 explored the possibility that the interference produced by phonologically similar distractors was not specifically associated with the attentional blink, but occurred even when only one target (T2) had to be identified. Because previous studies showed that, if T1 and T2 are defined by the same feature (color) ignoring T1 still produces an attentional blink (Chun, 1997; Spalek, Falcon, \& Di Lollo, 2006), all white T1s were converted to black and, for target similar trials, replaced by a letter dissimilar to T2 and to the critical distractors. Consequently, sequences contained only one white T2. Critical distractors varied in similarity and occupied the same positions relative to the white $\mathrm{T} 2$, as in the previous experiments.

\section{Method \\ Subjects. Thirty-three new students from the same pool participated. \\ Stimulus Materials, Apparatus, and Procedure. The condi- tions were unchanged, except that only one white target, the former $\mathrm{T} 2$, was reported. \\ Results and Discussion \\ Target identification was performed with a high level of accuracy and did not differ significantly across condi- tions: The means were $92 \%$ in the dissimilar distractor condition, $91 \%$ in the similar distractor condition (differ- ent from "T2"), and 91\% in the distractor condition (simi- lar to "T2"). This also applied to subjects whose scores were below the median. These results indicate that similar distractors do not interfere with target detection per se, but that they specifically impair conditional T2 $\mid \mathrm{T} 1$ report.}

\section{EXPERIMENT 4}

Does phonological similarity of distractors affect $\mathrm{T} 2 \mid \mathrm{T} 1$ report only when target location is unpredictable, thus prompting a high level of distractor processing? This possibility was examined by presenting $\mathrm{T} 1$ and $\mathrm{T} 2$ in the same fixed lag 3 serial positions.

\author{
Method \\ Subjects. Subjects from Experiment 3 participated with task \\ order counterbalanced.
}

Stimulus Materials, Apparatus, and Procedure. Only target and distractor similarity was manipulated with T1 in third and T2 in sixth position in every sequence, and subjects were informed about this. Otherwise, the conditions were the same as before.

\section{Results and Discussion}

Conditional T2 $\mid \mathrm{T} 1$ scores were subjected to an ANOVA and to a planned contrast that indicated no significant difference between Condition 1 (targets and distractors all similar to one another) and Condition 2 (targets similar and distractors similar, with similarity differing between them). After exclusion of Condition 1, a 2 (target similarity) $\times 2$ (distractor similarity) ANOVA was performed. Only distractor similarity affected performance: T2 $\mid \mathrm{T} 1$ report was worse when distractors were similar (51.5\%) than in the dissimilar condition $[59.3 \% ; F(1,32)=9.74$, $\left.M S_{\mathrm{e}}=204.4, p<.005\right]$.

These results confirmed the finding that the presence of phonologically similar distractors impairs dual target report. This was not due to uncertainty of target location, because targets were always in the same serial positions. Consequently, phonological similarity of distractors specifically impairs dual target identification and does not interfere with single target identification. Thus, phonological encoding is initiated during or after T1 identification for subsequent items that do not require it.

Perhaps the involuntary phonological encoding for irrelevant items occurs only when targets and distractors belong to the same category-namely, letters. Could phonological processing be inhibited for items from a nontarget category? This possibility was explored in Experiment 5 by changing the target category; targets were digits, whereas distractors were letters, a task that yields a reliable attentional blink (Chun \& Potter, 1995).

\section{EXPERIMENT 5}

In Experiment 5, two-digit targets were randomly selected on each trial, and distractors were the letters previously used. Thus, targets were phonologically dissimilar and were dissimilar to distractors both categorically and phonologically. Could distractor interference more readily be inhibited if targets require activation and search for instances of a different taxonomic category - namely, Arabic numerals? Abstract letter identities are a component in theories of word recognition (see, e.g., M. Coltheart, Rastle, Perry, Langdon, \& Ziegler, 2001). Numbers (e.g., $1,2,3)$ are not represented in the letter recognition system. Neuropsychological evidence of double dissociations between written letter recognition, word recognition, and Arabic number processing supports this distinction (Cohen, Dehaene, Chochon, Lehéricy, \& Naccache, 2000). So the category difference between letters and numbers is important, because their visual/orthographic representations are assumed to be in separate detector systems. Additionally, the phonological form of number and letter names differs, 
and this should reduce interference between their phonological representations.

\section{Method}

Subjects. Forty-four new students from the same pool participated.

Stimulus Materials. The digits 2-9, T1 and T2, (printed in the same black font and size as the letters) were sampled without replacement and presented at lags 1,2,3, and 5. (Pilot testing indicated that white digits among black letters were easily detected with negligible attentional blink.) Distractors were similar and dissimilar letters from Experiments 1-4 (excluding ambiguous I and O).

Apparatus and Procedure. These were unchanged, except that instructions defined targets as digits.

\section{Results and Discussion}

The percentages of correct T2 $\mid \mathrm{T} 1$ reports were calculated and subjected to an ANOVA with lag $(1,2,3,5)$ and distractor similarity (similar, dissimilar) as withinsubjects factors. The mean scores are shown in Figure 3. There was a significant effect of lag $[F(3,129)=32.65$, $\left.M S_{\mathrm{e}}=281.5, p<.0001\right]$. Trend analysis indicated significant linear $[F(1,129)=23.50, p<.0001]$ and quadratic $[F(1,129)=74.25, p<.0001]$ components. As Figure 3 demonstrates, there was substantial lag 1 sparing and an attentional blink. Performance was significantly lower with similar distractors than with dissimilar ones $\left[F(1,43)=14.98, M S_{\mathrm{e}}=1149.4, p<.0005\right]$. Planned contrasts demonstrated that distractor similarity significantly impaired performance only at lags $2[F(1,129)=$ $5.18, p<.05]$ and $3[F(1,129)=14.25, p<.0005]$.

Thus, phonologically similar distractors reduced target report at lags 2 and 3, increasing the magnitude of the attentional blink, even when targets and distractors belonged to different categories. Consequently, there was no evidence suggesting that phonological coding of distractors could be suppressed when targets belonged to another category, despite the fact that the phonological forms of target and distractor names are unrelated.

\section{GENERAL DISCUSSION}

In the dual target paradigm, distractors are irrelevant, and subjects therefore need not and should not encode them in any way. However, previous research has shown that distractors are encoded semantically in this task. Maki, Frigen, and Paulson (1997) found that a semantically related distractor preceding $\mathrm{T} 2$ reduced the attentional blink. Presumably this occurs because semantic access is automatic, and the effect was to reduce the deficit. The results of our experiments demonstrated that distractors are also encoded phonologically in these tasks. This too presumably reflects automaticity, but, unlike the semantic effects, the consequences are negative. If targets are to be reported, they must be phonologically recoded, so a phonological recoding processor is initiated for T1. It appears that subjects are unable flexibly to switch this processor on and off rapidly after it is engaged by T1. Consequently, the phonological processor encodes post-T1 distractors as well, even when distractors and targets belong to different semantic categories (e.g., digits vs. letters). The repetition of similar phonological features disrupts or possibly overwrites encoding of T2. Alternatively, repetition of similar letter names might lead to a form of "attentional capture" induced by similar phonological features (Maki, 2006, personal communication). Although theories of the attentional blink assume that distractors between $\mathrm{T} 1$ and $\mathrm{T} 2$ interfere with target processing, they do not specifically predict the additional impairment produced by distractor phonological similarity.

However, evidence of obligatory phonological recoding is pervasive in silent reading, provoking errors and slowing responses in sentence evaluation (V. Coltheart, Laxon, Rickard, \& Elton, 1988), semantic categorization of single words (V. Coltheart, Patterson, \& Leahy, 1994) and in Stroop tasks (M. Coltheart, Woollams, Kinoshita, \& Perry, 1999).

The results reported here provide evidence for rapid early activation of phonological codes during RSVP dual target identification and indicate that interference caused by similar irrelevant items specifically occurs when a second target has to be identified soon after the first. Detection of the first target initiates explicit phonological encoding in addition to target registration in VSTM. If no other target follows, consolidation is possible without

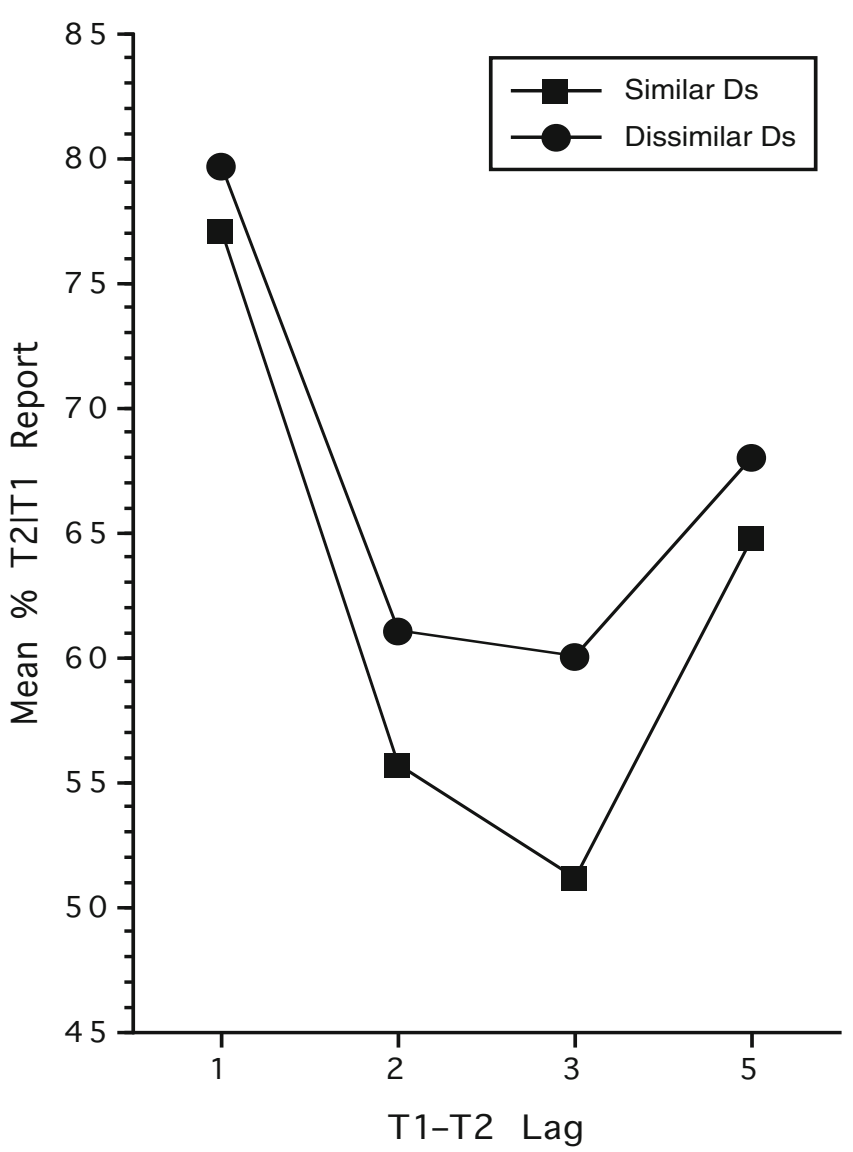

Figure 3. Mean percentages of correct $\mathrm{T} 2 \mid \mathrm{T} 1$ report for the similar distractor and dissimilar distractor conditions as a function of T1-T2 lag for Experiment 5. 
disruption by distractor phonological similarity, as observed with single target identification in Experiment 3. However, if the limited capacity consolidation process must maintain the first target and then be engaged for a second target, subjects have inadequate control over the phonological recoding process: This process should be, but cannot be, disabled when subsequent incoming stimuli are irrelevant distractors.

\section{AUTHOR NOTE}

This research was supported by an ARC Centre Grant to the Macquarie Centre for Cognitive Science (MACCS), Macquarie University, Australia. We thank Max Coltheart for invaluable discussion and comments, and we gratefully acknowledge support from CIMec, Polo di Rovereto, University of Trento, Italy, during preparation of this article. Correspondence concerning this article should be addressed to V. Coltheart, Macquarie Centre for Cognitive Science, Macquarie University, NSW 2109, Australia (e-mail: veronika@maccs.mq.edu.au).

\section{REFERENCES}

Bates, T. C., \& D'Oliveiro, L. (2003). PsyScript: A Macintosh application for scripting experiments. Behavior Research Methods, Instruments, \& Computers, 35, 565-576.

Chun, M. M. (1997). Temporal binding errors are redistributed in the attentional blink. Perception \& Psychophysics, 59, 1191-1199.

Chun, M. M., \& PotTer, M. C. (1995). A two-stage model for multiple target detection in rapid serial visual presentation. Journal of Experimental Psychology: Human Perception \& Performance, 21, 109-127.

Cohen, L., Dehaene, S., Chochon, F., Lehéricy, S., \& Naccache, L. (2000). Language and calculation within the parietal lobe: A combined cognitive, anatomical and fMRI study. Neuropsychologia, 38, 1426-1440.

Coltheart, M., Rastle, K., Perry, C., Langdon, R., \& Ziegler, J. (2001). DRC: A dual route cascaded model of visual word recognition and reading aloud. Psychological Review, 108, 204-256.

Coltheart, M., Woollams, A., Kinoshita, S., \& Perry, C. (1999). A position-sensitive Stroop effect: Further evidence for a left-to-right component in print-to-speech conversion. Psychonomic Bulletin \& Review, 6, 456-463.

Coltheart, V., \& Langdon, R. (1998). Recall of short word lists presented visually at fast rates: Effects of phonological similarity and word length. Memory \& Cognition, 26, 330-342.
Coltheart, V., Laxon, V., Rickard, M., \& Elton, C. (1988). Phonological recoding in reading for meaning by adults and children. Journal of Experimental Psychology: Learning, Memory, \& Cognition, 14, 387-397.

Coltheart, V., Patterson K., \& Leahy, J. (1994). When a ROWS is a ROSE: Phonological effects in written word comprehension. Quarterly Journal of Experimental Psychology, 47A, 917-955.

ConRad, R., \& Hull, A. J. (1964). Input modality, acoustic confusion and memory span. British Journal of Psychology, 55, 429-432.

Di Lollo, V., Kawahara, J., Ghorashi, S. M. S., \& EnNs, J. T. (2005). The attentional blink: Resource depletion or temporary loss of control? Psychological Research, 69, 191-200.

Dux, P. E., \& Coltheart, V. (2005). The meaning of the mask matters: Evidence of conceptual interference in the attentional blink. Psychological Science, 16, 775-779.

IsaAK, M. I., Shapiro, K. L., \& Martin, J. (1999). The attentional blink reflects retrieval competition among multiple rapid serial visual presentation items: Tests of an interference model. Journal of Experimental Psychology: Human Perception \& Performance, 25, 1774-1792

Maki, W. S., Bussard, G., Lopez, K, \& Digby, B. (2003). Sources of interference in the attentional blink: Target-distractor similarity revisited. Perception \& Psychophysics, 65, 188-201.

Maki, W. S., Frigen, K., \& PAulson, K. (1997). Associative priming by targets and distractors during rapid serial visual presentation: Does word meaning survive the attentional blink? Journal of Experimental Psychology: Human Perception \& Performance, 23, 1014-1034.

Olson, I. R., Chun, M. M., \& Anderson, A. K. (2001). Effects of phonological length on the attentional blink for words. Journal of Experimental Psychology: Human Perception \& Performance, 27, 1116-1123.

Raymond, J. E., Shapiro, K. L., \& Arnell, K. M. (1992). Temporary suppression of visual processing in an RSVP task: An attentional blink? Journal of Experimental Psychology: Human Perception \& Performance, 18, 849-860.

Seiffert, A. E., \& Di Lollo, V. (1997). Low-level masking in the attentional blink. Journal of Experimental Psychology: Human Perception \& Performance, 23, 1061-1073.

Spalek, T. M., Falcon, L. J., \& Di Lollo, V. (2006). Attentional blink and attentional capture: Endogenous versus exogenous control over paying attention to two important events in close succession. Perception \& Psychophysics, 68, 674-684.

(Manuscript received May 11, 2006; revision accepted for publication November 30, 2006.) 